\title{
Self-Optimized Catalysts: Hot-Electron Driven Photosynthesis of Catalytic Photocathodes
}

\author{
Evgenia Kontoleta, ${ }^{\S}{ }^{\circledR}$ Sven H. C. Askes, ${ }^{\S}$ and Erik C. Garnett*() \\ Center for Nanophotonics, AMOLF, Science Park 104, 1098 XG Amsterdam, Netherlands \\ Supporting Information
}

\begin{abstract}
Photogenerated hot electrons from plasmonic nanostructures are very promising for photocatalysis, mostly due to their potential for enhanced chemical selectivity. Here, we present a self-optimized fabrication method of plasmonic photocathodes using hot-electron chemistry, for enhanced photocatalytic efficiencies. Plasmonic $\mathrm{Au} / \mathrm{TiO}_{2}$ nanoislands are excited at their surface plasmon resonance to generate hot electrons in an aqueous bath containing a platinum (cocatalyst) precursor. Hot electrons drive the deposition of $\mathrm{Pt}$ cocatalyst nanoparticles, without any nanoparticle functionalization and negligible applied bias, close to the hotspots of the

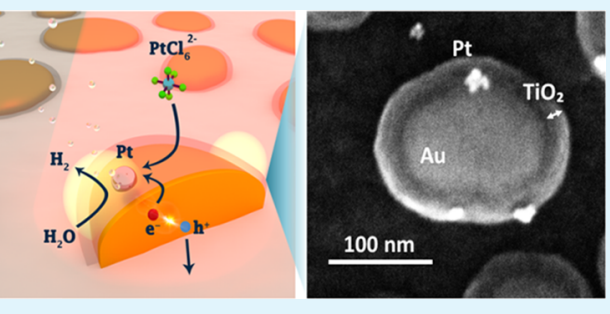
plasmonic nanoislands. The presence of $\mathrm{TiO}_{2}$ is crucial for achieving higher chemical reaction rates. The $\mathrm{Au} / \mathrm{TiO}_{2} / \mathrm{Pt}$ photocathodes synthesized using hot-electron chemistry show a photocatalytic activity of up to 2 times higher than that of a control made with random electrodeposited Pt nanoparticles. This light-driven positioning of the cocatalyst close to the same positions where hot electrons are most efficiently generated and transferred represents a novel and simple method for synthesizing complex, self-optimized photocatalytic nanostructures with improved efficiency and selectivity.
\end{abstract}

KEYWORDS: hot-electron chemistry, plasmonic nanoparticles, photoelectrochemistry, photocatalysis, Au/TiO , Pt photodeposition

\section{INTRODUCTION}

Plasmonic nanoparticles can offer alternative pathways for driving chemical reactions compared to common semiconductors. The light-controlled selectivity over chemical products on plasmonic nanoparticles makes them interesting for photocatalysis. ${ }^{1-4}$ The different reactivity from standard thermal catalysis is thought to arise from highly energetic, "hot" electron-hole pairs formed upon plasmonic excitation and decay. $^{5-7}$ Excitation of the plasmonic nanoparticle at the resonant wavelength results in the collective coherent oscillation of conduction band electrons with a concomitant enhancement of the electric field at specific hotspots. At these hotspots, the plasmon excitation can decay via Landau damping and thereby excites a single electron to create a highly-energetic electronhole pair, which is initially out of thermal equilibrium with the surroundings, that is, "hot" ${ }^{8-10}$ Hot carriers from plasmonic nanoparticles have so far been used for the reduction of chemisorbed $p$-nitrothiophenol ${ }^{9}$ or aryl diazonium salts, ${ }^{11}$ oxidation of citrate, ${ }^{12}$ hydrogen production, ${ }^{13-16}$ and conversion of $\mathrm{CO}_{2}$ to formic acid. ${ }^{1}$

Plasmon-driven photoelectrochemical reactions seem very promising, but the recorded efficiencies are still too low for practical use. This low efficiency is mostly due to the short hotcarrier lifetime (<3 ps for Au or Ag nanoparticles) ${ }^{18-20}$ Higher efficiencies have been reached by bringing the metal nanostructures in contact with an appropriately chosen semiconducting layer, allowing for rapid hot-electron extraction and reduced recombination via a Schottky barrier., 16,19,21-25 Similarly, hot holes can be extracted with a hole-transfer layer such as nickel oxide, ${ }^{15}$ leaving the electrons on the structure.
However, hot-carrier extraction alone is often not sufficient; cocatalytic nanoparticles are necessary to achieve high efficiencies in almost all photoelectrochemical processes. Ideally, this cocatalyst has the lowest possible loading, both to reduce the amount of costly precious metals such as platinum and rhodium as well as minimize reflection and parasitic absorption of incident light. Furthermore, it should be optimum to localize the cocatalyst only near the plasmonic hotspots where hot electrons are generated most efficiently and catalysis is driven most favorably. ${ }^{26-29}$ Nevertheless, there are few practical, low-cost, and efficient techniques to position a cocatalyst with such nanoscale precision. For instance, Mubeen et al. ${ }^{16}$ demonstrated fully autonomous plasmonic water splitting with a Au nanorod device with two individually deposited cocatalysts. However, the fabrication techniques dictated the localization of both cocatalysts, which do not necessarily imply an optimal spatial configuration, that is, the hotspots and cocatalyst sites are potentially mismatched.

Here, we propose a self-optimized fabrication strategy where hot electrons are used both for the localized deposition of cocatalyst nanoparticles and the subsequent photocatalysis (Figure 1). Our approach removes the need for lithographic patterning of either the plasmonic nanoparticles or cocatalyst while simultaneously allowing for the ideal spatial distribution at any given loading. We used random Au plasmonic nanoislands on ITO coated with $\mathrm{TiO}_{2}$ as the plasmonically active substrate.

Received: June 21, 2019

Accepted: September 2, 2019

Published: September 2, 2019 


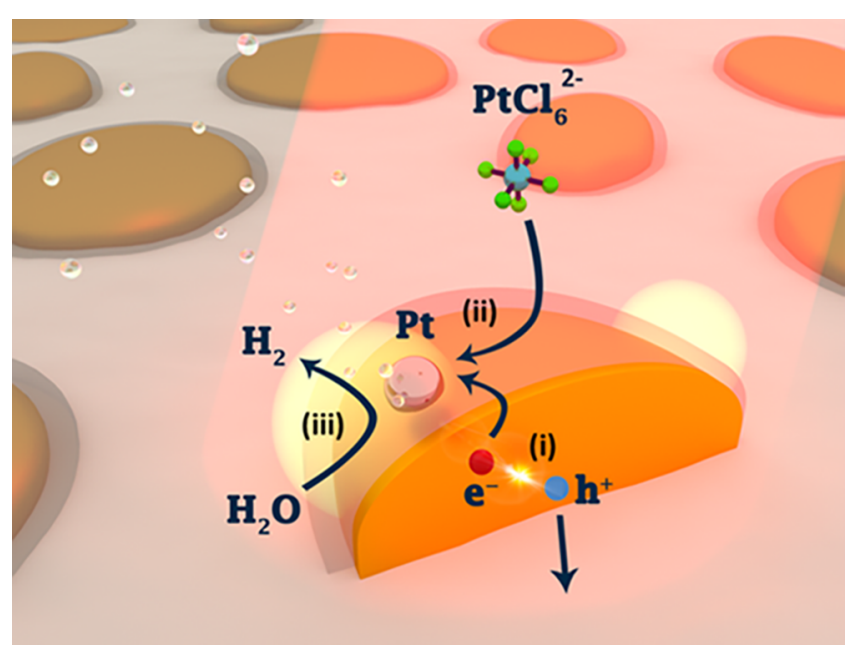

Figure 1. Schematic illustration of the self-optimized catalysts. (i) Hot electrons and hot holes are created in $\mathrm{Au} / \mathrm{TiO}_{2}$ nanoislands after excitation at $638 \mathrm{~nm}$. (ii) Hot electrons reduce hexachloroplatinate at the plasmonic hotspots (light yellow areas) to form platinum nanoparticles (cocatalyst). (iii) Hot electrons reach the newly formed platinum nanoparticles and accelerate hydrogen production at the same excitation wavelength.

Both the deposition of Pt cocatalyst nanoparticles from solution and the subsequent photocatalysis were conducted under 638 $\mathrm{nm}$ illumination (within the plasmonic absorption peak). By comparing the photocatalytic performance of our self-optimized photodeposition method to that from random electrodeposition (with identical loading and dark current characteristics), we see up to a factor of 2 increase in the efficiency of photocatalysis.

\section{EXPERIMENTAL SECTION}

General Procedures. Chemicals were purchased from major chemical suppliers and used as received. Sample characterization was done with scanning electron microscopy (FEI Verios 460, acceleration beam voltage $5 \mathrm{kV}$, and beam current $100 \mathrm{pA}$ ), X-ray diffraction (Bruker $\mathrm{D} 2$ Phaser, $\mathrm{Cu} \mathrm{K} \alpha$ radiation, and wavelength $=1.5418 \AA$ ), and atomic force microscopy (Veeco Dimension 3100 AFM). X-ray photoemission spectroscopy (XPS) was performed in a home-built ultrahigh vacuum chamber, operating at a base pressure of below $5 \times 10^{-9} \mathrm{mbar}$. A $\mathrm{XM} 1200$ monochromatic X-ray source ( $\mathrm{Al} \alpha$-K line, Scienta Omicron) was used for X-ray excitation of the sample under a $45^{\circ}$ angle. Photoemitted electrons were collected using a HIPP-3 analyzer (Scienta Omicron). Spectra were charge corrected using the binding energy of $1 \mathrm{~s}$ carbon $(284.8 \mathrm{eV})$. A UV/Vis/NIR spectrophotometer (PerkinElmer, L750) was used for acquiring the absorbance spectra.

Preparation of Au Nanoislands. Plasmonic Au nanoislands were prepared on ITO-covered glass slides (Figure S1) following a literature procedure. ${ }^{15} 15 \times 15 \mathrm{~mm}$ indium tin oxide (ITO) substrates $(150 \mathrm{~nm}$ on glass) were cleaned with detergent, rinsed with demineralized water, acetone, and isopropanol, and dried in a stream of $\mathrm{N}_{2}$. The substrates were placed in a sputter coater (Leica EM ACE600) where $8 \mathrm{~nm}$ of Au was sputtered with a rate of $\sim 0.35 \mathrm{~nm} / \mathrm{s}$ and measured with a quartz crystal thickness monitor during sputtering. After deposition of the thin Au layer, the samples were placed in a tube oven, which was brought to $300{ }^{\circ} \mathrm{C}$ with a rate of $9.2^{\circ} \mathrm{C} / \mathrm{min}$, kept at constant temperature for $1 \mathrm{~h}$, and subsequently allowed to cool down to room temperature.

Atomic Layer Deposition of $\mathrm{TiO}_{2}$ on Au Nanoislands. Atomiclayer deposition (ALD) of $\mathrm{TiO}_{2}$ on Au nanoislands was conducted in a custom thermal ALD system developed in-house at $100{ }^{\circ} \mathrm{C}$. The base pressure of the system was $0.03-0.07 \mathrm{mbar}$, and during deposition, the pressure was kept at $1.1 \mathrm{mbar}$ with an influx of $\mathrm{N}_{2}$. Each cycle of $\mathrm{TiO}_{2}$ deposition consisted of injecting a $10 \mathrm{~ms}$ pulse of $\mathrm{TiCl}_{4}$ vapor, waiting for $18 \mathrm{~s}$, injecting a $10 \mathrm{~ms}$ pulse of $\mathrm{H}_{2} \mathrm{O}$ vapor, and then waiting for another 18 s. The deposition rate was $0.4 \AA$ per cycle. The $\mathrm{TiO}_{2}$ thickness on $\mathrm{Au}$ nanoislands was estimated by depositing $\mathrm{TiO}_{2}$ in parallel to a $\mathrm{Si}$ substrate and measuring the layer thickness by ellipsometry using a dielectric model of $\mathrm{TiO}_{2}$ and native $\mathrm{SiO}_{2}$ on a $\mathrm{Si}$ substrate. Typically, 300 cycles of $\mathrm{TiO}_{2}(18 \mathrm{~nm})$ were deposited on the $\mathrm{Au}$ nanoislands. For the purpose of electrical connection (see below), a $2 \mathrm{~mm}$ strip of the substrates was masked with Kapton tape during ALD.

Photoelectrodeposition of $\mathrm{Pt}$ on $\mathrm{Au} / \mathrm{TiO}_{2}$ Nanoislands. Photoelectrodeposition of platinum on $\mathrm{Au} / \mathrm{TiO}_{2}$ nanoisland substrates was conducted in a three-electrode photoelectrochemical cell (PEC) connected to a potentiostat (Biologic, SP-200) (Figure S2). The Au/ $\mathrm{TiO}_{2}$ substrate (working electrode) was electrically connected to the potentiostat with a conductive aluminum tape (Advance Tapes AT521) on the top $2 \mathrm{~mm}$ of the substrate. A rubber ring $(\varnothing 8 \mathrm{~mm})$ was placed centrally on the $\mathrm{Au} / \mathrm{TiO}_{2}$ working electrode, and this assembly was clamped tightly on a $6 \mathrm{~mm}$ hole on the outside of the electrochemical cell. Only the central $6 \mathrm{~mm}$ was thus in contact with the electrolyte. A leakless miniature $\mathrm{Ag} / \mathrm{AgCl}$ electrode (Mengel Engineering EDET072) was used as a reference electrode, and a Pt wire was used as a counter electrode. The electrolyte was prepared with $100 \mathrm{mM}$ $\mathrm{Na}_{2} \mathrm{SO}_{4}$ and $4 \mathrm{mM} \mathrm{H} \mathrm{H}_{2} \mathrm{PtCl}_{6}$ in MilliQ water $(18.2 \mathrm{M} \Omega \cdot \mathrm{cm})$ and adjusted to $\mathrm{pH} 3.0-3.4$ with aliquots of $\mathrm{NaOH}(2 \mathrm{M})$. The electrolyte was prepared at least $48 \mathrm{~h}$ in advance to allow hydrolysis of $\mathrm{H}_{2} \mathrm{PtCl}_{6}$ which has been found important for the deposition of $\mathrm{Pt}$ on $\mathrm{TiO}_{2}{ }^{30}$ The PEC was filled with an $8 \mathrm{~mL}$ electrolyte, and the sample was illuminated from the back without interacting first with the electrolyte with a laser beam $\left(0.5 \mathrm{~W} / \mathrm{cm}^{2}\right)$. Meanwhile, the potential of the working electrode was kept constant (chronoamperometry mode), typically at $+0.25 \mathrm{~V}$ versus $\mathrm{Ag} / \mathrm{AgCl}$. After photoelectrodeposition, samples were rinsed with $\mathrm{H}_{2} \mathrm{O}$ and dried with $\mathrm{N}_{2}$.

Wavelength Dependence Measurements. Wavelength dependence measurements on $\mathrm{Au} / \mathrm{TiO}_{2}$ photocathodes were conducted in the presence of hexachloroplatinate under the same conditions as the photoelectrodeposition of $\mathrm{Pt}$ using now a different light source (supercontinuum laser, Fianium WL-SC-390-3), which was made monochromatic using an acousto-optical tunable filter (AOTF-Crystal Technologies). The signal was readout from the potentiostat (Biologic, SP-200) after lock-in amplification (Stanford Research Systems SR830), while the transmission of the AOTF was digitally modulated at $70 \mathrm{~Hz}$. The potential of the working electrode was kept constant (chronoamperometry mode) at $+0.25 \mathrm{~V}$ versus $\mathrm{Ag} / \mathrm{AgCl}$. The current measurement was started in the dark (beam was blocked manually), and every $20 \mathrm{~s}$, it was alternated between a different excitation wavelength in the dark.

Electrodeposition of Pt Nanoparticles. Random deposition of platinum nanoparticles was performed on the $\mathrm{Au} / \mathrm{TiO}_{2}$ photocathodes in the presence of hexachloroplatinate $\left(\mathrm{H}_{2} \mathrm{PtCl}_{6}, \mathrm{pH} 3\right)$, using a pulsed electrodeposition method introduced by Liu et al. ${ }^{31}$ Specifically, we used differential pulsed amperometry to control the sequence and the duration of the pulses sent to the sample. In the absence of light, first, a $-0.6 \mathrm{~V}$ versus RHE potential was applied to the sample for $5 \mathrm{~s}$. In sequence, the potential was changed to $0.6 \mathrm{~V}$ versus RHE and kept at this value for $25 \mathrm{~s}$. This total period of $30 \mathrm{~s}$ is considered one cycle, and the amount of deposited $\mathrm{Pt}$ was controlled by the number of cycles.

Photoconditioning. During this step, $-0.25 \mathrm{~V}$ versus RHE was applied to the $\mathrm{Pt} / \mathrm{Au} / \mathrm{TiO}_{2}$ samples in the three-electrode photoelectrochemical cell in the presence of a deoxygenated (purged for $1 \mathrm{~h}$ with $\mathrm{N}_{2}$ ) phosphate buffer $(\mathrm{pH} 7,0.1 \mathrm{M})$. The duration of the photoconditioning step was $45 \mathrm{~s}$; in the first $20 \mathrm{~s}$, the sample was kept in the dark and followed by $25 \mathrm{~s}$ of illumination with a $638 \mathrm{~nm}$ beam $(0.5$ $\left.\mathrm{W} / \mathrm{cm}^{2}\right)$.

Photocatalysis Measurements on $\mathrm{Au} / \mathrm{TiO}_{2} / \mathrm{Pt}$ Photocathodes. Photocatalysis measurements of the $\mathrm{Au} / \mathrm{TiO}_{2} / \mathrm{Pt}$ photocathodes were conducted in the same three-electrode photoelectrochemical cell as the photoelectrodeposition of Pt nanoparticles but with a different electrolyte (phosphate buffer, $\mathrm{pH} 7$, purged with nitrogen for $1 \mathrm{~h}$ ). The current flow from the samples was measured as a function of the potential with a scan rate of $20 \mathrm{mV} / \mathrm{s}$, under $638 \mathrm{~nm}\left(0.5 \mathrm{~W} / \mathrm{cm}^{2}\right)$ chopped illumination, with a chopping frequency of $25 \mathrm{~Hz}$. 
FDTD Simulations of $\mathrm{Au} / \mathrm{TiO}_{2}$ Nanoislands. Finite difference time domain (FDTD) simulations were performed using the $3 \mathrm{D}$ Maxwell solver software package Lumerical. An AFM height map of $\mathrm{Au} / \mathrm{TiO}_{2}$ nanoislands $(2 \times 2 \mu \mathrm{m})$ was imported as a surface, which functioned as the $\mathrm{TiO}_{2}$ layer. Optical constants retrieved by ellipsometry (Figure S3) were used for this layer. The bare Au nanoislands layer was retrieved from the same AFM height map by importing it in the $3 \mathrm{D}$ simulation program blender and using the displacement modifier algorithm to mimic the subtraction of the $18 \mathrm{~nm}$ conformal $\mathrm{TiO}_{2}$ layer. The resulting $\mathrm{Au}$ nanoisland height map was exported, interpolated to uniform $x-y$ spacing using Origin software, and imported in Lumerical as a surface to function as the Au layer. Finally, an ITO substrate was added to complete the sample geometry. The background index was water $(n=1.33)$. The simulation space additionally consisted of a $2.3 \times 2.3 \times 0.5 \mu \mathrm{m}(1 \times \mathrm{w} \times \mathrm{h})$ FDTD box with perfectly matched layer boundary conditions and a $4 \times 4 \mathrm{~nm}$ mesh size, a $2.1 \times 2.1 \times 0.4 \mu \mathrm{m}$ plane wave source at $638 \mathrm{~nm}$, and a $1.8 \times 1.8 \times$ $0.2 \mu \mathrm{m}$ advanced power absorption monitor. Here, the power absorption is proportional to the electric field intensity and imaginary part of the permittivity and is given in the fraction of absorbed power per cubic meter $\left(P_{\mathrm{abs}} / \mathrm{m}^{3}\right)$.

Inductively Coupled Plasma Mass Spectrometry Measurements. The preparation of the samples for the inductively coupled plasma mass spectrometry (ICP-MS) measurements took place via the transfer of the glass $/ \mathrm{ITO} / \mathrm{Au} / \mathrm{TiO}_{2} / \mathrm{Pt}$ samples in the liquid phase. Each sample was immersed in $1 \mathrm{~mL}$ of an aqua regia solution, $\mathrm{HNO}_{3}$ ( $\geq 65 \%$, puriss. p.a.): $\mathrm{HCl}(37 \%)(1: 3)$, for $5.5 \mathrm{~h}$ at $60^{\circ} \mathrm{C}$ until the glass substrate was completely transparent. The aqua regia solution was then left to cool down overnight. The transfer of the solution was being done carefully to a centrifuge tube where it was further diluted with MilliQ water $(18.2 \mathrm{M} \Omega \cdot \mathrm{cm})$ in a total volume of $10 \mathrm{~mL}$.

\section{RESULTS AND DISCUSSION}

Plasmonic Au nanoislands were prepared on ITO-covered glass slides with the aim of using them as absorbing platforms for the selective hot-electron driven deposition of the cocatalytic nanoparticles. It has already been shown that this type of sample is photocatalytic and can also support hotspots. ${ }^{15,32}$ Briefly, an $8 \mathrm{~nm}$ gold film was sputtered coated on ITO substrates and subsequently annealed at $300{ }^{\circ} \mathrm{C}$ for $1 \mathrm{~h}$ to spontaneously form $\mathrm{Au}$ nanoislands (Figure 2a). The nanoislands were coated with a thin $\mathrm{TiO}_{2}$ layer with atomic layer deposition (ALD), which acts as a hot-electron filter prohibiting recombination of the photogenerated charges in the metal by providing a high density of electron-accepting states. ${ }^{33} \mathrm{X}$-ray diffraction (XRD) showed that the $\mathrm{TiO}_{2}$ layer had crystallized in the anatase phase (Figure S4) after annealing, which has been shown to be favorable for accepting hot electrons compared to rutile or amorphous $\mathrm{TiO}_{2} \cdot{ }^{34}$ SEM images (Figure 2a) and AFM maps (Figure $2 \mathrm{~b}$ ) showed final structures $\left(\mathrm{Au} / \mathrm{TiO}_{2}\right)$ with a mean largest diameter of $151 \pm 56 \mathrm{~nm}$ and a mean height of $44 \pm$ $8 \mathrm{~nm}$ (Figure S5). Overlays of secondary electron and backscattered electron SEM images (Figure 2c and Figure S6) revealed the presence of a $18 \pm 2 \mathrm{~nm} \mathrm{TiO}_{2}$ layer around the gold nanoislands, and the thickness of which was further confirmed by size distribution measurements before and after the ALD step (Figure 2d and Figure S7) and ellipsometry data (Figure S3). The chosen $\mathrm{TiO}_{2}$ thickness arose from preliminary results for optimization of the photocurrent of our system (see below).

Absorption spectra showed a strong plasmonic peak of the $\mathrm{Au} / \mathrm{TiO}_{2}$ structures centered between 640 and $700 \mathrm{~nm}$, depending on small sample thickness differences (Figure 2e), which is an ideal wavelength range for the generation of hot electrons, as plasmonic excitations below $600 \mathrm{~nm}$ are strongly dampened by interband transitions in bulk Au. ${ }^{35,36}$ The size distribution of the nanostructures could strongly affect the a

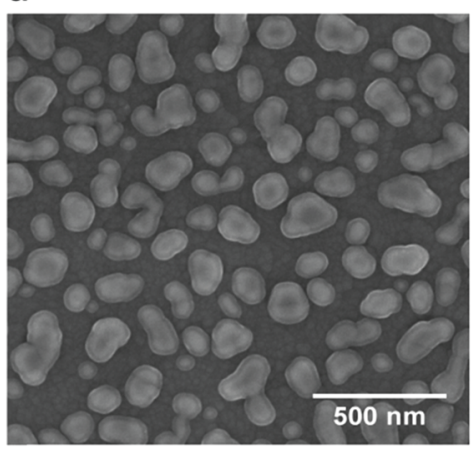

b

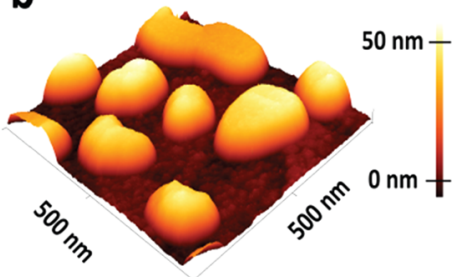

C

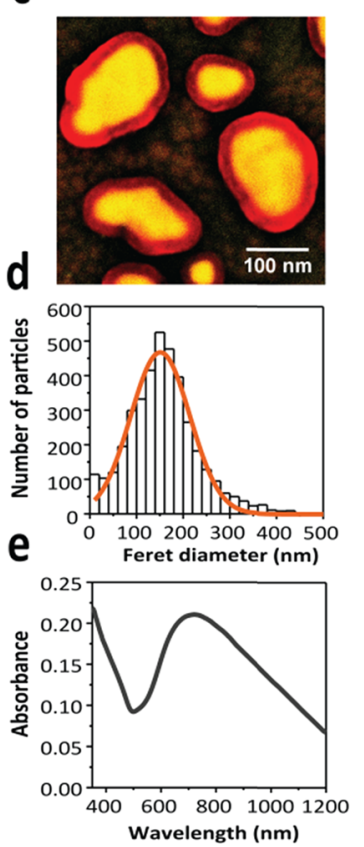

Figure 2. Characterization of $\mathrm{Au} / \mathrm{TiO}_{2}$ nanoislands on ITO/glass substrate. (a) SEM image, (b) AFM map, (c) overlay of backscattered (yellow) and secondary electron (red) images where the strong contrast between $\mathrm{TiO}_{2}$ and $\mathrm{Au}$ nanoislands originates from the lack of electron backscatter by the lighter elements of the $\mathrm{TiO}_{2}$ shell, (d) size distribution of $\mathrm{Au} / \mathrm{TiO}_{2}$ nanoislands after SEM imaging analysis with a fitting Gaussian curve (orange solid line), and (e) absorbance spectrum.

surface plasmon resonance of the final samples and could be easily tuned by altering the thickness of the sputter-coated $\mathrm{Au}$ film. The size distribution chosen here gave a resonance far from the interband transition wavelength region but still in a high electron energy range. A redshift and a broadening of the absorption peak of the $\mathrm{Au}$ nanoislands were observed (Figure S8) after the addition of $\mathrm{TiO}_{2}$, which can be attributed to the higher embedding refractive index and possibly also to increased interparticle coupling. ${ }^{32,37}$ The $\mathrm{TiO}_{2}$ layer has negligible optical absorption at $638 \mathrm{~nm}$ with an onset of band gap absorption in the ultraviolet, as expected (Figure S3). When deposited on ITO, there is some parasitic absorption, but this is far below $(\sim 9$ times) than that of the plasmonic nanoislands (Figure S8) and does not contribute significantly to photocurrent (Figure S9). $\mathrm{The} \mathrm{Au} / \mathrm{TiO}_{2}$ nanoislands were easily prepared on a bulk scale and were suitable for studying hot-electron photoreactions with red light.

The ability of $\mathrm{Au} / \mathrm{TiO}_{2}$ nanoislands to generate hot $\mathrm{e}^{-}$, which can drive a chemical reaction for the synthesis of cocatalytic nanoparticles, was investigated. The nanoislands were excited by a $638 \mathrm{~nm}$ laser beam $\left(0.5 \mathrm{~W} / \mathrm{cm}^{2}\right)$ in a three-electrode photoelectrochemical cell under a potentiostatic control using a $\mathrm{Ag} / \mathrm{AgCl}$ reference electrode. The current flow from the sample (working electrode) to a platinum wire (counter electrode) was recorded in the presence of an aqueous platinum precursor $\left(0.04 \mathrm{mM} \mathrm{H}_{2} \mathrm{PtCl}_{6}\right.$ in $0.1 \mathrm{M} \mathrm{Na}_{2} \mathrm{SO}_{4}, \mathrm{pH} \mathrm{3}$, and deoxygenated). At open-circuit voltage conditions ( $\mathrm{OCV}=$ $470-580 \mathrm{mV}$ vs RHE), a very little photocurrent was observed, but sweeping the potential to more negative values increased the Pt photodeposition rate (Figure S9c). Figure 3a shows a typical current density versus time curve of the $\mathrm{Au} / \mathrm{TiO}_{2}$ nanoislands both under $638 \mathrm{~nm}$ light illumination (laser on) and in the dark 

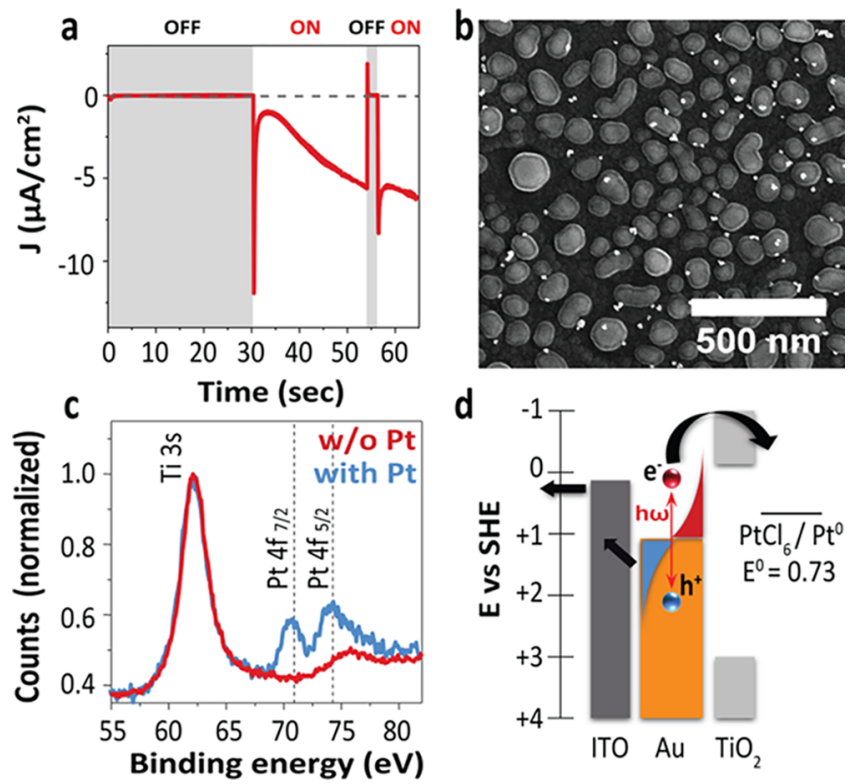

Figure 3. (a) Current density vs time plot of $\mathrm{Au} / \mathrm{TiO}_{2}$ nanoislands in presence of hexachloroplatinate $\left(\mathrm{pH} \mathrm{3}, 0.04 \mathrm{mM}\right.$ in $0.1 \mathrm{M} \mathrm{Na}_{2} \mathrm{SO}_{4}$ aqueous solution, and $450 \mathrm{mV}$ vs RHE). (b) SEM image of $\mathrm{Pt}$ nanoparticles on $\mathrm{Au} / \mathrm{TiO}_{2}$ nanoislands after illumination of the latter at $638 \mathrm{~nm}\left(0.5 \mathrm{~W} / \mathrm{cm}^{2}\right)$ in hexachloroplatinate and recording of $125 \mu \mathrm{C} /$ $\mathrm{cm}^{2}$ of electrical charge from the sample. (c) X-ray photoemission spectrum of the samples before (light blue line) and after photoelectrodeposition of $\mathrm{Pt}$ nanoparticles (red line). Dashed lines indicate literature values for metallic $\mathrm{Pt} 4 \mathrm{f}_{7 / 2}$ and $4 \mathrm{f}_{5 / 2}$ binding energies. $^{44}$ (d) Simplified band diagram of $\mathrm{Au} / \mathrm{TiO}_{2}$ nanoislands on ITO and flow of hot electrons and holes during the photoelectrodeposition process.

(laser off) at $450 \mathrm{mV}$ versus RHE. While the dark current was negligible throughout the whole measurement, the photocurrent gradually increased as a function of time $(t=30-55$ and 55-65 s, Figure 3a). Furthermore, almost no photocurrent $(\sim 16 \mathrm{nA} /$ $\mathrm{cm}^{2}, 40-1000$ times lower than $\mathrm{Au} / \mathrm{TiO}_{2}$ samples) was recorded from bare $\mathrm{TiO}_{2}$ (Figure S9), indicating that the photoactivity comes from plasmonic absorption in the $\mathrm{Au}$ and hot-electron photoreduction. In our approach, current measurements are retrieved from the whole photoelectrode, a bulk method that has already been used as evidence for hot-electron flow. ${ }^{15,16,38}$ Single-nanoparticle photocurrent measurements have also confirmed that the presence of photocurrent at the surface plasmon resonance of the nanostructure is related to the flow of hot carriers. ${ }^{39}$

SEM images showed that after an electrical charge of $125 \mu \mathrm{C} /$ $\mathrm{cm}^{2}$ was passed to the sample, new nanoparticles appeared on the $\mathrm{Au} / \mathrm{TiO}_{2}$ samples (bright clusters, Figure 3b). The newly formed nanoparticles corresponded to a mixture of $\mathrm{Pt}(\mathrm{II}) /$ $\mathrm{Pt}(\mathrm{IV})$ salts/oxides according to XPS (Figure S10) and EDX measurements (Figure S11), so they were further reduced by a "photoconditioning" step and using again hot-electron chemistry. During this step, the platinum-containing electrolyte was exchanged with an aqueous phosphate buffer to avoid further deposition of $\mathrm{Pt}$ species, and the sample was irradiated for $25 \mathrm{~s}$ at $638 \mathrm{~nm}\left(0.5 \mathrm{~W} / \mathrm{cm}^{2}\right)$ at an applied potential of $-250 \mathrm{mV}$ versus RHE (Figure S12). After the photoconditioning procedure, the observed platinum $4 f_{7 / 2}$ and $4 f_{5 / 2}$ binding energy peaks matched a metallic Pt reference (Figure $3 \mathrm{c}$ ), indicating that the reduced particles consisted mainly of metallic platinum, favorable for catalyzing many chemical reactions. ${ }^{40}$ The fact that the initial photoreaction did not produce any metallic Pt may be ascribed to $\mathrm{Pt}^{4+}$ kinetically outcompeting $\mathrm{Pt}^{2+}$ species for hot electrons. However, photoconditioning at the same potential as the initial photodeposition step $(450 \mathrm{mV})$ did not result in $\mathrm{Pt}^{0}$ nanoparticles. This indicates that it is the extra potential is crucial for the full reduction of $\mathrm{Pt}$ species. Apparently, hot electrons that have been injected in the $\mathrm{TiO}_{2}$ conduction band do not possess sufficient reducing power to fully reduce hexachloroplatinate to metallic platinum. These results mirror those from $\mathrm{Xi}$ et al. ${ }^{30}$ who have shown that the electrochemical reduction of hexachloroplatinate leads to mixed valence materials. More reducing potentials (than $-250 \mathrm{mV}$ vs RHE) were avoided during the photoconditioning step, because then, the distinction between hot electron mechanism and bare electrochemical reduction of Pt species would be difficult. As a result, the photocurrent measured in the $\mathrm{Au} / \mathrm{TiO}_{2}$ nanoislands could eventually be correlated with the deposition of $\mathrm{Pt}$ nanoparticles.

We thus examined the correlation of the photocurrent generation (i.e., the hot-electron production) with the absorptance of the Au plasmonic nanoislands. Au nanoislands coated with $\mathrm{TiO}_{2}$ were excited at different wavelengths (440$760 \mathrm{~nm}$, with $20 \mathrm{~nm}$ step) with a supercontinuum laser, while the current was recorded (Figure S13) in the presence of hexachloroplatinate. The incident photon to current efficiency (IPCE) was calculated (see the Supplementary Information for more information) for every excitation wavelength and plotted together with the absorptance spectrum of the same sample as a function of the wavelength (Figure S14). The results show that there is a good correlation between the absorptance of the $\mathrm{Au}$ nanoislands and the IPCE values, except for in the blue region (440-500 nm). Seemingly, photogenerated electrons resulting from interband transitions (high energy electrons) barely participate in the photoreduction of hexachloroplatinate. This is reasonable since these electrons are excited from $\mathrm{d}$ band levels to energy levels close to or below the Fermi level of $\mathrm{Au} .{ }^{19}$ These electrons therefore do not have enough energy to surpass the $\mathrm{Au} / \mathrm{TiO}_{2}$ Schottky barrier and contribute to the generation of photocurrents. So, apparently only hot electrons with energy high enough to be injected to the conduction band of $\mathrm{TiO}_{2}$ can reach the hexachloroplatinate molecules and reduce them to $\mathrm{Pt}$ species.

The plasmonic excitation of Au nanoislands clearly leads to Pt nanoparticle formation, but to further confirm that this occurs via a hot-electron mechanism, we have conducted several control experiments. The most obvious alternative explanation is a simple heating effect. The large increase in photocurrent after the addition of $\mathrm{TiO}_{2}$ (Figure 3a and Figure S9) as well as the correlation between the absorptance and IPCE spectra (Figure S14) already point toward a hot electron rather than a thermal mechanism. Hot electrons with energy high enough to overcome the Schottky barrier are transferred into the $\mathrm{TiO}_{2}$ layer where the barrier reduces the chances of back transfer and recombination, increasing the efficiency of $\mathrm{Pt}$ formation (Figure $3 \mathrm{~d}$ ). The improved yield of $\mathrm{Pt}$ with the addition of $\mathrm{TiO}_{2}$ alone cannot completely rule out a thermal effect since hexachloroplatinate molecules bind much better to the $\mathrm{TiO}_{2}$ surface than on $\mathrm{Au}$, which allows for suitable electron acceptors being always present as the hot electron arrives at the $\mathrm{TiO}_{2} / \mathrm{H}_{2} \mathrm{O}$ interface. ${ }^{34}$ However, the better surface binding cannot explain the correlation between the absorption and IPCE spectra described above. Additionally, the photocurrent showed a linear dependence on the laser intensity over a range of two orders of magnitude (Figure S15), consistent with a hot-electron 
mechanism and inconsistent with laser heating. ${ }^{7,38}$ The temperature on the surface of the sample was measured with a FLIR thermal camera (Figure S16) during irradiation at $638 \mathrm{~nm}$ $\left(0.5 \mathrm{~W} / \mathrm{cm}^{2}\right)$ and reached up to $30{ }^{\circ} \mathrm{C}$. As a follow-up control test, we conducted dark heating experiments and saw no $\mathrm{Pt}$ nanoparticle formation at $40^{\circ} \mathrm{C}$ even after $20 \mathrm{~min}$ (Figure S17), showing that thermal decomposition is not playing a role in our experiments.

The observed photocurrent density increase as a function of time during the photoelectrodeposition of $\mathrm{Pt}$ on the $\mathrm{Au} / \mathrm{TiO}_{2}$ nanoislands (Figure 3a) can be explained by the presence of the first atoms of $\mathrm{Pt}$ formed on the surface of $\mathrm{TiO}_{2}$ acting as electron sinks and enhancing the reaction rate. ${ }^{41}$ The sudden current transient ("spikes" at 30 and around 55 s, Figure 3a) every time the laser is switched on could be the result of charge recombination at the electrode/electrolyte interface. As a function of time, the amplitude of the current transients seems decreased $(\sim 55 \mathrm{sec})$, and this could be correlated with the presence of the first $\mathrm{Pt}$ atoms, which reduce the charge recombination and improve the separation of the hot electrons from the hot holes. ${ }^{42}$ The correlation between plasmonic hotspots and Pt deposition sites was unfortunately very challenging to be investigated because the hotspots were not very well defined. In addition, FDTD simulations of the threedimensional AFM-deduced structures (without Pt deposits) showed that the hotspots on this type of nanostructures are generally spread out across large distances around the nanoparticle edges (Figures S18 and S19). In Figure 3b, some $\mathrm{Pt}$ nanoparticles form in areas between the $\mathrm{Au}$ nanoislands where no plasmonic hotspots appear according to FDTD simulations, and the enhancement of the absorbed power is mostly observed on the Au nanoislands (Figures S18 and S19). This indicates that hot electrons injected into the $\mathrm{TiO}_{2}$ layer can diffuse and form $\mathrm{Pt}$ nanoparticles that somewhat removed from the plasmonic hotspots, in contrast with previous reports where $\mathrm{TiO}_{2}$ was not used. ${ }^{43}$ Nevertheless, even in the case that hot electrons are reaching the electrolyte and reducing hexachloroplatinate further from the actual hotspots, the positioning of the cocatalyst can be still considered as optimum for the photocathode as a whole. Hot electrons are created and then diffused until they meet a site that supports an efficient transfer to the photoelectrode surface. After further illumination of the $\mathrm{Au} / \mathrm{TiO}_{2} / \mathrm{Pt}$ photocathodes, the new photogenerated hot electrons will probably follow the same path as the first time to the electrolyte but now meeting the cocatalyst nanoparticles first. In that way, the whole process is not limited by the exact characteristics of the $\mathrm{TiO}_{2}$ film but enables any imperfections "work" in its favor.

To illustrate the photocatalytic behavior of the hot-electron driven prepared photocathodes $\left(\mathrm{Au} / \mathrm{TiO}_{2} / \mathrm{Pt}_{\text {photo }}\right)$, we performed current density versus potential (CV) scans in the presence of an aqueous phosphate buffer $(0.1 \mathrm{M}, \mathrm{pH} 7$, and deoxygenated). The current flow of the $\mathrm{Au} / \mathrm{TiO}_{2} / \mathrm{Pt}_{\text {photo }}$ samples was recorded under chopped illumination at $638 \mathrm{~nm}$ while sweeping the electrochemical potential of the working electrode to more negative (reducing) values (Figure 4a,b, blue solid line). The CV scan shows an increase in the current density under illumination (laser on), which becomes higher toward more negative potentials. The dark current (laser off) remains negligible ( $\sim 130$ times lower than the photocurrent) until an applied potential of around $-0.35 \mathrm{~V}$ versus RHE where it starts increasing rapidly. The difference between onset potential of photo and dark current can be explained by the Schottky barrier
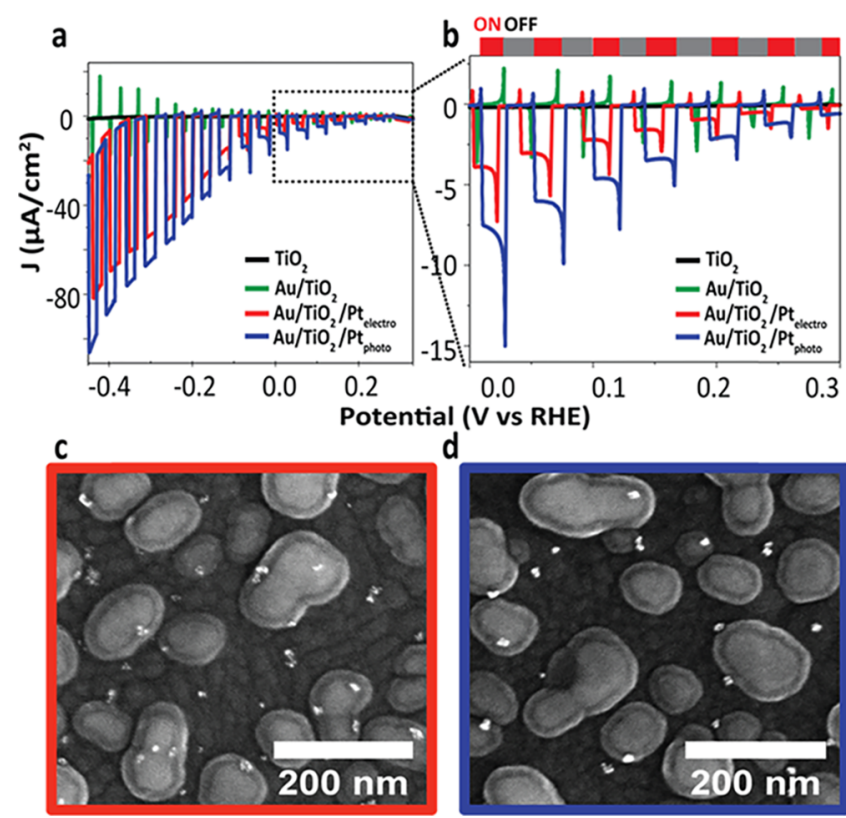

Figure 4. (a) Current density vs potential plots of $\mathrm{TiO}_{2}$ (black solid line), $\mathrm{Au} / \mathrm{TiO}_{2}$ nanoislands (green solid line), $\mathrm{Au} / \mathrm{TiO}_{2}$ nanoislands with randomly electrodeposited $\mathrm{Pt}$ nanoparticles $\left(\mathrm{Au} / \mathrm{TiO} 2 / \mathrm{Pt}_{\text {electro, }}\right.$, solid red line), and localized photoelectrodeposited $\mathrm{Pt}\left(\mathrm{Au} / \mathrm{TiO}_{2} /\right.$ $\mathrm{Pt}_{\text {photo }}$, solid blue line) in $\mathrm{pH} 7$ phosphate buffer under chopped $638 \mathrm{~nm}$ illumination $\left(0.5 \mathrm{~W} / \mathrm{cm}^{2}\right)$. (b) Zoom-in of plot (a) at low applied potentials $\left(0.0-0.3 \mathrm{~V}\right.$ vs RHE). (c, d) SEM images of $\mathrm{Au} / \mathrm{TiO}_{2} / \mathrm{Pt}_{\text {electro }}$ and $\mathrm{Au} / \mathrm{TiO}_{2} / \mathrm{Pt}_{\text {photo }}$ photocathodes, respectively.

between $\mathrm{Au}$ and $\mathrm{TiO}_{2}$. The more negative the applied potential, the smaller the $\mathrm{Au} / \mathrm{TiO}_{2}$ Schottky barrier due to the shift of the Fermi level of $\mathrm{Au}$ toward the vacuum level and the conduction band of $\mathrm{TiO}_{2} \cdot{ }^{45}$ In the absence of light, the $\mathrm{Au} / \mathrm{TiO}_{2}$ Schottky barrier does not allow electrons to flow from $\mathrm{Au}$ to $\mathrm{TiO}_{2}$ and drives the chemical reaction, so almost no current is recorded until the Fermi level of Au equalizes with the conduction band of $\mathrm{TiO}_{2}$ at $-0.3 \mathrm{~V}$ versus RHE. Assuming a Fermi-Dirac hotelectron distribution in the $\mathrm{Au}$ nanoparticles under illumination, ${ }^{5}$ more electrons will have high enough energy for transfer to the $\mathrm{TiO}_{2}$, increasing the photocurrent. Control measurements were also conducted on bare $\mathrm{TiO}_{2}$ as well as $\mathrm{Au} / \mathrm{TiO}_{2}$ to investigate performance without the cocatalyst and plasmonic light absorber, respectively. CV scans on both of these configurations (Figure 4a,b, black and green solid lines, respectively) revealed that the recorded photocurrent was $\sim 1000$ and $\sim 30$ times lower, for $\mathrm{TiO}_{2}(\sim 4 \mathrm{nA})$ and $\mathrm{Au} / \mathrm{TiO}_{2}$ $(140 \mathrm{nA})$, respectively, than those for the $\mathrm{Au} / \mathrm{TiO}_{2} / \mathrm{Pt}_{\text {photo }}$ samples at $0 \mathrm{~V}$ versus RHE. This proves that both the plasmonic absorber and cocatalyst play key roles in the photocatalytic behavior. Considering the potential onset of the photoreduction reaction and the species available in solution, water reduction to hydrogen is the most likely photochemical reaction product.

To test the self-optimized behavior of our approach, we investigated if our method of preparing photocathodes with hotelectron injection performs better in photocatalysis than that of a common electrodeposition method of depositing the cocatalyst (see the Supporting Information for more details) under the same conditions. To warrant a valid comparison, we carefully controlled the electrodeposition conditions to achieve samples with an identical amount of platinum, with the same morphology and oxidation state, verified by ICP-MS, XPS, and SEM (Table S2, Figure S20, and Figure 4c,d). CV scans (Figure 
$4 a, b$, blue and red solid lines) showed that when we let the plasmonic nanostructures decide where the cocatalyst will be deposited (i.e., hot-electron deposition, $\mathrm{Au} / \mathrm{TiO}_{2} / \mathrm{Pt}_{\text {photo }}$ ), the photocurrent density was higher (up to 2 times) than when the cocatalyst was randomly electrodeposited $\left(\mathrm{Au} / \mathrm{TiO}_{2} / \mathrm{Pt}_{\text {electro }}\right)$, especially at small applied potentials. This suggests that the localization of the cocatalyst close to the hotspots of the plasmonic photocathodes, where the hot electrons reach the photocathode/electrolyte interface, promotes the chemical reaction. The positioning of the cocatalyst exactly on the pathway of the photogenerated hot electrons toward the electrolyte could contribute to the better utilization of the hot electrons participating in the chemical reaction. In case of a random cocatalyst distribution on the plasmonic photocathodes, the hot electrons will have to diffuse further than their original path to find the cocatalyst. During this additional diffusion, the probability of their recombination with the respective hot holes, remaining in the gold, is increased. The potential dependence of the photocurrent varied somewhat from sample to sample (Figure 4a, Figures S21 and S22), suggesting that the hotelectron energy distribution is sensitive to the exact $\mathrm{Au}$ nanoisland geometry and/or differences on the $\mathrm{TiO}_{2}$ surface. Therefore, only samples, which had exactly the same amount of deposited platinum (retrieved from XPS, Figures S20, S21, and S22 and ICP-MS data, and Table S2) and the same dark current (see CV scans for photoelectrodeposited and electrodeposited samples, Figures S21 and S22) were compared. Further work trying to use this potential distribution to map out hot-electron energy distributions is ongoing.

\section{CONCLUSIONS}

In summary, we demonstrate a lithography-free method for creating plasmonic photocathodes with cocatalyst nanoparticles placed selectively at hot-electron generation sites. The plasmonic hotspots on $\mathrm{Au} / \mathrm{TiO}_{2}$ nanoislands were used both to localize the $\mathrm{Pt}$ nanoparticle cocatalyst and to do photocatalysis. The presence of $\mathrm{TiO}_{2}$ proved to be essential for reducing recombination of hot carriers and led to higher photocurrent values. Besides providing a good hot-electron filter, the $\mathrm{TiO}_{2}$ may also enhance binding of the Pt precursor molecules on its surface. We also showed that photocathodes with a cocatalyst deposited using hot electrons have better photocatalytic performance than those made with randomly placed electrodeposited Pt. This self-optimized photoelectrode strategy, where plasmonic nanostructures themselves determine the cocatalyst position, is a very promising route for simple fabrication of complex photocatalytic nanostructures that could lead to enhanced plasmonic solar fuel production.

\section{ASSOCIATED CONTENT}

\section{S Supporting Information}

The Supporting Information is available free of charge on the ACS Publications website at DOI: 10.1021/acsami.9b10913.

Description of the ICPE calculation as well as extra experiments and characterization (ICP-MS, EDX, XRD, ellipsometry, and XPS) of the samples and FDTD simulation setup (PDF)

\section{AUTHOR INFORMATION}

\section{Corresponding Author}

*E-mail: E.Garnett@amolf.nl.

\section{ORCID}

Evgenia Kontoleta: 0000-0002-3327-1523

Sven H. C. Askes: 0000-0001-6538-3645

Erik C. Garnett: 0000-0002-9158-8326

Author Contributions

${ }^{\S}$ E.K. and S.A. contributed equally.

\section{Notes}

The authors declare no competing financial interest.

\section{ACKNOWLEDGMENTS}

We thank Reinout Jaarsma (Advanced Research Center for Nanolithography: ARCNL institute) for his technical assistance with the XPS measurements, Jan Zomerdijk (AMOLF institute) for his help with the electrical measurements, and Peter Nobels (Chemical Biological Soil Laboratory, Wageningen University and Research) for the conduction of the ICP-MS measurements. We also acknowledge Dr. Andrea Baldi (Dutch Institute for Fundamental Energy Research) and Dr. Marco Valenti (AMOLF) for the useful discussions. This work is part of the research program of the Foundation for Fundamental Research on Matter (FOM), which is financially supported by the Netherlands Foundation for Scientific Research (NWO).

\section{REFERENCES}

(1) Zhang, X.; Li, X.; Zhang, D.; Su, N. Q.; Yang, W.; Everitt, H. O.; Liu, J. Product Selectivity in Plasmonic Photocatalysis for Carbon Dioxide Hydrogenation. Nat. Commun. 2017, 8, 14542.

(2) Linic, S.; Aslam, U.; Boerigter, C.; Morabito, M. Photochemical Transformations on Plasmonic Metal Nanoparticles. Nat. Mater. 2015, 14, 567-576.

(3) Marimuthu, A.; Zhang, J.; Linic, S. Tuning Selectivity in Propylene Epoxidation by Plasmon Mediated Photo-Switching of $\mathrm{Cu}$ Oxidation State. Science 2013, 339, 1590-1593.

(4) Quiroz, J.; Barbosa, E. C. M.; Araujo, T. P.; Fiorio, J. L.; Wang, Y. C.; Zou, Y. C.; Mou, T.; Alves, T. V.; De Oliveira, D. C.; Wang, B.; Haigh, S. J.; Rossi, L. M.; Camargo, P. H. C. Controlling Reaction Selectivity over Hybrid Plasmonic Nanocatalysts. Nano Lett. 2018, 18, 7289-7297.

(5) Boriskina, S. V.; Ghasemi, H.; Chen, G. Plasmonic Materials for Energy: From Physics to Applications. Mater. Today 2013, 16, 375386.

(6) Wang, P.; Huang, B.; Dai, Y.; Whangbo, M.-H. Plasmonic Photocatalysts: Harvesting Visible Light with Noble Metal Nanoparticles. Phys. Chem. Chem. Phys. 2012, 14, 9813-9825.

(7) Christopher, P.; Xin, H.; Marimuthu, A.; Linic, S. Singular Characteristics and Unique Chemical Bond Activation Mechanisms of Photocatalytic Reactions on Plasmonic Nanostructures. Nat. Mater. 2012, 11, 1044-1050.

(8) Clavero, C. Plasmon-Induced Hot-Electron Generation at Nanoparticle/Metal-Oxide Interfaces for Photovoltaic and Photocatalytic Devices. Nat. Photonics 2014, 8, 95-103.

(9) Cortés, E.; Xie, W.; Cambiasso, J.; Jermyn, A. S.; Sundararaman, R.; Narang, P.; Schlücker, S.; Maier, S. A. Plasmonic Hot Electron Transport Drives Nano-Localized Chemistry. Nat. Commun. 2017, 8, 14880 .

(10) Forcherio, G. T.; Baker, D. R.; Boltersdorf, J.; Leff, A. C.; McClure, J. P.; Grew, K. N.; Lundgren, C. A. Targeted Deposition of Platinum onto Gold Nanorods by Plasmonic Hot Electrons. J. Phys. Chem. C 2018, 122, 28901-28909.

(11) Nguyen, V. Q.; Ai, Y.; Martin, P.; Lacroix, J. C. Plasmon-Induced Nanolocalized Reduction of Diazonium Salts. ACS Omega 2017, 2, 1947-1955.

(12) Schlather, A. E.; Manjavacas, A.; Lauchner, A.; Marangoni, V. S.; DeSantis, C. J.; Nordlander, P.; Halas, N. J. Hot Hole Photoelectrochemistry on Au@SiO2@Au Nanoparticles. J.Phys. Chem. Lett. 2017, 8, 2060-2067. 
(13) Wang, F.; Wong, R. J.; Ho, J. H.; Jiang, Y.; Amal, R. Sensitization of $\mathrm{Pt} / \mathrm{TiO}_{2}$ Using Plasmonic Au Nanoparticles for Hydrogen Evolution under Visible-Light Irradiation. ACS Appl. Mater. Interfaces 2017, 9, 30575-30582.

(14) Wan, D. Y.; Zhao, Y. L.; Cai, Y.; Asmara, T. C.; Huang, Z.; Chen, J. Q.; Hong, J.; Yin, S. M.; Nelson, C. T.; Motapothula, M. R.; Yan, B. X.; Xiang, D.; Chi, X.; Zheng, H.; Chen, W.; Xu, R.; Ariando; Rusydi, A.; Minor, A. M.; Breese, M. B. H.; Sherburne, M.; Asta, M.; Xu, Q.-H.; Venkatesan, T. Electron Transport and Visible Light Absorption in a Plasmonic Photocatalyst Based on Strontium Niobate. Nat. Commun. 2017, 8, 15070.

(15) Robatjazi, H.; Bahauddin, S. M.; Doiron, C.; Thomann, I. Direct Plasmon-Driven Photoelectrocatalysis. Nano Lett. 2015, 15, 61556161.

(16) Mubeen, S.; Lee, J.; Singh, N.; Krämer, S.; Stucky, G. D.; Moskovits, M. An Autonomous Photosynthetic Device in Which All Charge Carriers Derive from Surface Plasmons. Nat. Nanotechnol. 2013, 8, 247-251.

(17) Kumar, D.; Lee, A.; Lee, T.; Lim, M.; Lim, D. K. Ultrafast and Efficient Transport of Hot Plasmonic Electrons by Graphene for Pt Free, Highly Efficient Visible-Light Responsive Photocatalyst. Nano Lett. 2016, 16, 1760-1767.

(18) Ahmadi, T. S.; Logunov, S. L.; El-Sayed, M. A. Picosecond Dynamics of Colloidal Gold Nanoparticles. J. Phys. Chem. 1996, 100, 8053-8056.

(19) Brongersma, M. L.; Halas, N. J.; Nordlander, P. PlasmonInduced Hot Carrier Science and Technology. Nat Nanotechnol. 2015, $10,25-34$.

(20) Zhang, Y.; He, S.; Guo, W.; Hu, Y.; Huang, J.; Mulcahy, J. R.; Wei, W. D. Surface-Plasmon-Driven Hot Electron Photochemistry. Chem. Rev. 2018, 118, 2927-2954.

(21) Valenti, M.; Venugopal, A.; Tordera, D.; Jonsson, M. P.; Biskos, G.; Schmidt-Ott, A.; Smith, W. A. Hot Carrier Generation and Extraction of Plasmonic Alloy Nanoparticles. ACS Photonics 2017, 4, $1146-1152$.

(22) Ma, X. C.; Dai, Y.; Yu, L.; Huang, B. B. Energy Transfer in Plasmonic Photocatalytic Composites. Light: Sci. Appl. 2016, 5, No. e16017.

(23) Giugni, A.; Torre, B.; Toma, A.; Francardi, M.; Malerba, M.; Alabastri, A.; Proietti Zaccaria, R.; Stockman, M. I.; Di Fabrizio, E. HotElectron Nanoscopy Using Adiabatic Compression of Surface Plasmons. Nat. Nanotechnol. 2013, 8, 845-852.

(24) Yu, Y.; Ji, Z.; Zu, S.; Du, B.; Kang, Y.; Li, Z.; Zhou, Z.; Shi, K.; Fang, Z. Ultrafast Plasmonic Hot Electron Transfer in Au Nanoantenna/ $\mathrm{MoS}_{2}$ Heterostructures. Adv. Funct. Mater. 2016, 26, 63946401.

(25) Shan, H.; Yu, Y.; Wang, X.; Luo, Y.; Zu, S.; Du, B.; Han, T.; Li, B.; Li, Y.; Wu, J.; Lin, F.; Shi, K.; Tay, B. K.; Liu, Z.; Zhu, X.; Fang, Z. Direct Observation of Ultrafast Plasmonic Hot Electron Transfer in the Strong Coupling Regime. Light: Sci. Appl. 2019, 8, 9.

(26) Sousa-Castillo, A.; Comesaña-Hermo, M.; Rodríguez-González, B.; Pérez-Lorenzo, M.; Wang, Z.; Kong, X. T.; Govorov, A. O.; CorreaDuarte, M. A. Boosting Hot Electron-Driven Photocatalysis through Anisotropic Plasmonic Nanoparticles with Hot Spots in $\mathrm{Au}-$ TiO2Nanoarchitectures. J. Phys. Chem. C 2016, 120, 11690-11699.

(27) Wu, B.; Liu, D.; Mubeen, S.; Chuong, T. T.; Moskovits, M.; Stucky, G. D. Anisotropic Growth of TiO2onto Gold Nanorods for Plasmon-Enhanced Hydrogen Production from Water Reduction. J. Am. Chem. Soc. 2016, 138, 1114-1117.

(28) Zheng, Z.; Tachikawa, T.; Majima, T. Single-Particle Study of PtModified Au Nanorods for Plasmon-Enhanced Hydrogen Generation in Visible to near-Infrared Region. J. Am. Chem. Soc. 2014, 136, 68706873.

(29) Ortiz, N.; Zoellner, B.; Hong, S. J.; Ji, Y.; Wang, T.; Liu, Y.; Maggard, P. A.; Wang, G. Harnessing Hot Electrons from Near IR Light for Hydrogen Production Using Pt-End-Capped-AuNRs. ACS Appl. Mater. Interfaces 2017, 9, 25962-25969.
(30) Xi, C.; Chen, Z.; Li, Q.; Jin, Z. Effects of $\mathrm{H}+, \mathrm{Cl}$ - and $\mathrm{CH} 3 \mathrm{COOH}$ on the Photocatalytic Conversion of $\mathrm{PtCl} 62$ - in Aqueous $\mathrm{TiO} 2$ Dispersion. J. Photochem. Photobiol., A 1995, 87, 249-255.

(31) Liu, Y.; Gokcen, D.; Bertocci, U.; Moffat, T. P. Self-Terminating Growth of Platinum Films by Electrochemical Deposition. Science 2012, 338, 1327-1330.

(32) Kang, M.; Park, S. G.; Jeong, K. H. Repeated Solid-State Dewetting of Thin Gold Films for Nanogap-Rich Plasmonic Nanoislands. Sci. Rep. 2015, 5, 14790.

(33) Lee, Y. K.; Lee, H.; Lee, C.; Hwang, E.; Park, J. Y. Hot-ElectronBased Solar Energy Conversion with Metal-Semiconductor Nanodiodes. J. Phys. Condens. Matter 2016, 28, 254006.

(34) Macyk, W.; Burgeth, G.; Kisch, H. Photoelectrochemical Properties of Platinum(IV) Chloride Surface Modified TiO2. Photochem. Photobiol. Sci. 2003, 2, 322-328.

(35) Lord, H. L.; Zhan, W.; Pawliszyn, J. Fundamentals and Applications of Needle Trap Devices: A Critical Review. Anal. Chim. Acta 2010, 677, 3.

(36) Kong, X.-T.; Wang, Z.; Govorov, A. O. Plasmonic Nanostars with Hot Spots for Efficient Generation of Hot Electrons under Solar Illumination. Adv. Opt. Mater. 2017, 5, 1-10.

(37) Yu, Y.; Wen, W.; Qian, X. Y.; Liu, J. B.; Wu, J. M. UV and Visible Light Photocatalytic Activity of $\mathrm{Au} / \mathrm{TiO} 2$ Nanoforests with Anatase/ Rutile Phase Junctions and Controlled Au Locations. Sci. Rep. 2017, 7, 41253.

(38) Zilio, P.; Dipalo, M.; Tantussi, F.; Messina, G. C.; De Angelis, F. Hot Electrons in Water: Injection and Ponderomotive Acceleration by Means of Plasmonic Nanoelectrodes. Light: Sci. Appl. 2017, 6, No. e17002.

(39) Lee, H.; Lee, H.; Park, J. Y. Direct Imaging of Surface PlasmonDriven Hot Electron Flux on the Au Nanoprism/TiO 2. Nano Lett. 2019, 19, 891-896.

(40) Yu, W.; Porosoff, M. D.; Chen, J. G. Review of Pt-Based Bimetallic Catalysis: From Model Surfaces to Supported Catalysts. Chem. Rev. 2012, 112, 5780-5817.

(41) Shuang, S.; Lv, R.; Xie, Z.; Zhang, Z. Surface Plasmon Enhanced Photocatalysis of Au/Pt-Decorated TiO2 Nanopillar Arrays. Sci. Rep. 2016, 6, 26670.

(42) Radecka, M.; Wierzbicka, M.; Komornicki, S.; Rekas, M. Influence of $\mathrm{Cr}$ on Photoelectrochemical Properties of $\mathrm{TiO} 2$ Thin Films. Phys. B 2004, 348, 160-168.

(43) Kim, N. H.; Meinhart, C. D.; Moskovits, M. Plasmon-Mediated Reduction of Aqueous Platinum Ions: The Competing Roles of Field Enhancement and Hot Charge Carriers. J. Phys. Chem. C 2016, 120, $6750-6755$.

(44) Santiago, D.; Rodríguez-Calero, G. G.; Palkar, A.; BarrazaJimenez, D.; Galvan, D. H.; Casillas, G.; Mayoral, A.; Jose-Yacamán, M.; Echegoyen, L.; Cabrera, C. R. Platinum Electrodeposition on Unsupported Carbon Nano-Onions. Langmuir 2012, 28, 1720217210 .

(45) Scanlon, M. D.; Peljo, P.; Méndez, M. A.; Smirnov, E.; Girault, H. H. Charging and Discharging at the Nanoscale: Fermi Level Equilibration of Metallic Nanoparticles. Chem. Sci. 2015, 2705-2720. 